\title{
The enhanced antitumor effects of biodegradable cationic heparin-polyethyleneimine nanogels delivering HSulf-1 gene combined with cisplatin on ovarian cancer
}

\author{
PING LIU, MALING GOU, TAO YI, XIAORONG QI, CHUAN XIE, SHENGTAO ZHOU, \\ HONGXIN DENG, YUQUAN WEI and XIAZHAO
}

\begin{abstract}
Department of Gynecology and Obstetrics, Key Laboratory of Obstetric and Gynecologic and Pediatric Diseases and Birth Defects of Ministry of Education, West China Second Hospital; State Key Laboratory of Biotherapy and Cancer Center, Sichuan University, Chengdu 610041, Sichuan, P.R. China
\end{abstract}

Received April 26, 2012; Accepted June 19, 2012

DOI: 10.3892/ijo.2012.1558

\begin{abstract}
HSulf-1 (heparan sulfate 6-O-endosulfatase 1), a commonly downregulated gene in the majority of ovarian cancer cell lines, has been identified to play an important role in regulating tumorigenesis. Our previous studies demonstrated that HSulf-1 could inhibit angiogenesis and tumorigenesis in vivo. The employment of polymeric nanoparticles to deliver functional gene holds much promise as an effective therapeutic strategy against ovarian cancer. To develop more effective therapy, in this study, we investigated the antitumor effect of heparin-polyethyleneimine (HPEI) nanogels delivering HSulf-1 combined with cisplatin (DDP) on ovarian cancer. Expression of HSulf-1 in vitro and in vivo was determined by reverse transcription polymerase chain reaction (RT-PCR) and western blot analysis. A SKOV3 intraperitoneal ovarian carcinomatosis model in nude mice was established to assess the antitumor efficacy. Mice were treated with NS, pEP/HPEI complexes, pHSulf-1/HPEI complexes, DDP or pHSulf-1/HPEI plus DDP, respectively. Intraperitoneal tumors were weighed. Antiangiogenic effect in vivo was evaluated by CD31 immunostaining and alginate-encapsulate tumor cell assay. Detection of the proliferative cells and apoptotic cells in tumor tissues were performed by Ki-67 staining and TUNEL assay. Stable expression of HSulf-1 was detected in the pHSulf-1/HPEI and pHSulf-1/HPEI plus DDP groups. The combination of pHSulf-1/HPEI complexes with DDP exhibited enhanced anti-
\end{abstract}

Correspondence to: Professor Xia Zhao, Department of Gynecology and Obstetrics, Key Laboratory of Obstetric and Gynecologic and Pediatric Diseases and Birth Defects of Ministry of Education, West China Second Hospital; State Key Laboratory of Biotherapy and Cancer Center, Sichuan University, No. 20, Section 3, South People's Road, Chengdu 610041, Sichuan, P.R. China

E-mail: xia-zhao@126.com

Key words: HSulf-1, combination therapy, heparin-polyethyleneimine, ovarian cancer, angiogenesis, apoptosis tumor activity, compared with the monotherapy of HSulf-1 or DDP alone $(\mathrm{P}<0.01)$. the combination therapy exerted significant antitumor activity through enhanced antiangiogenesis, induction of apoptosis and suppression of cell proliferation. Collectively, these observations provide evidence that HPEI nanogels delivering HSulf- 1 combined with DDP may have a promising application in the therapy of human ovarian cancer.

\section{Introduction}

The full-length cDNA encoding human protein of extracellular heparan sulfate 6-O-endosulfatase 1, named HSulf-1, has been recently identified and characterized $(1,2)$. HSulf- 1 has a unique structural feature, enzymatic activity and signaling function which could selectively remove 6-O-sulfate from heparan sulfate proteoglycans (HSPGs) $(3,4)$. Since HSPGs serve as co-receptors for numerous heparin-binding growth factors, cytokines, chemokines and adhesion molecules, they could act as key regulator of growth factor and cell signaling in the extracellular matrix and on the cell surface $(5,6)$. HSulf- 1 could change HSPG-related signaling pathways, such as heparinbinding epidermal growth factor (HB-EGF), fibroblast growth factor-2 (FGF-2), hepatocyte growth factor (HGF), and vascular endothelial growth factor (VEGF). Therefore, dysregulation of HSulf-1 may exert significant effect on cell growth, angiogenesis and tumorigenesis (7-14). Previous studies have identified HSulf-1 as a downregulated gene in some tumor types including ovarian cancer, hepatocellular cancer, and head and neck squamous cell carcinoma $(7,8)$. Re-expression of HSulf-1 diminished heparin-binding growth factor signaling, inhibited cell proliferation, migration, invasion, angiogenesis and promoted drug-induced apoptosis in vitro (7-9) and in vivo (10-12).

Ovarian cancer is the leading cause of death from gynecological malignancies and accounts for $4 \%$ of all cancer types in American women (15). Despite improved methods of surgery, chemotherapy, radiotherapy and new biological therapies, the mortality in women with advanced, persistent or recurrent ovarian cancer still remains at a high level (16). Cisplatin (DDP), one of the most important chemotherapy drugs, has been widely used for the treatment of ovarian carcinoma. Despite the 
significant efficacy at treating ovarian cancer, cisplatin still has many problems in clinical use, such as severe side effects and resistance to the drug which undermine the curative potential of cisplatin. The antitumor mechanisms of DDP involve its capability to form bifunctional DNA crosslinks, failure to repair damaged DNA, cell cycle arrest, interference with DNA replication and the subsequent induction of cell death through apoptosis, necrosis, or both (17-20). The expression of HSulf-1 is repressed in the majority $(\sim 75 \%)$ of tumor tissues originated from ovarian cancer patients (7). In an effort to develop more effective and novel therapeutic approaches to combat ovarian cancer, we sought to develop combination therapy of HSulf-1 with DDP to treat ovarian cancer.

The application of gene therapy in cancer treatment has now been widely recognized, but the clinical employment of gene therapy is restricted mainly due to the lack of safe and efficient gene delivery technologies (21-24). At present, cationic polyethyleneimine (PEI) has been used as one of the most effective non-viral gene transfection agents. However, PEI is not biodegradable and its application has been compromised mainly due to the correlation of cytotoxicity, transfection efficiency and its chain length $(25,26)$. Intensive research has been carried out to couple short PEI chains into a longer one using biodegradable linkers to overcome this issue (27-30). In our previous study, the low molecular weight PEI was chemically conjugated into biodegradable cationic nanogels by heparin and HPEI could serve as a safe and efficient non-viral gene vector (31).

In the present study, we used HPEI nanogels delivering HSulf-1 combined with DDP to investigate the antitumor efficiency of combination therapy on human ovarian cancer. HSulf-1 exhibited significant capability of inhibiting tumor growth by way of reducing angiogenesis, decreasing cell proliferation and inducing apoptosis. The antitumor efficacy of HSulf-1 was enhanced when combined with DDP. Additionally, HPEI nanogels displayed efficient transfection without any conspicuous toxicity.

\section{Materials and methods}

Cell culture. The human ovarian epithelial serous cystadenocarcinoma cell line SKOV3, obtained from American Type Culture Collection (ATCC, Manassas, VA) was cultured in Dulbecco's modified Eagle's medium (DMEM) containing 10\% heat-inactivated fetal bovine serum (FBS), $2 \mathrm{mM} \mathrm{L}$-glutamine, $0.1 \mathrm{mg} / \mathrm{ml}$ of streptomycin and $100 \mathrm{U} / \mathrm{ml}$ of penicillin. The SKOV3 cells were incubated in a humidified atmosphere containing $5 \% \mathrm{CO}_{2}$ at $37^{\circ} \mathrm{C}$ and passaged every 3 days at a split ratio of $1: 3$ using trypsin.

Construction and purification of plasmid. The pVAX plasmid (Invitrogen, Carlsbad, CA) expressing HSulf-1 (GenBank accession number NM-001128205.1 GI:189571640) named pHSulf-1, was constructed in our laboratory as previously mentioned (32). The pVAX plasmid without HSulf-1 cDNA was used as an empty vector (named pEP). A broad scale preparation of plasmid DNA was purified using an EndoFree Plasmid Giga kit (Qiagen, Chatsworth, CA). The purified plasmids were eventually dissolved in Tris-EDTA buffer, stored at $-20^{\circ} \mathrm{C}$ for further use. The recombinant pHSulf-1 was confirmed by restriction digestion and DNA sequencing.
Synthesis of HPEI nanogels. The biodegradable cationic nanogel-HPEI were synthesized at the State Key Laboratory of Biotherapy and Cancer Center as previously described (31). Briefly, $50 \mathrm{mg}$ heparin was dissolved in $100 \mathrm{ml}$ 2-N-morpholino ethanesulfonic acid (MES) buffer solution $(0.05 \mathrm{M})$, and then, to activate the carboxylic acid groups of heparin, $20 \mathrm{mg}$ 1-ethyl-3-3-dimethylaminopropyl carbodiimide (EDC) and $30 \mathrm{mg}$ N-hydroxysuccinimide (NHS) were added into the solution. The solution was dropped into $20 \mathrm{ml}$ PEI2K solution $(7.5 \mathrm{mg} / \mathrm{ml})$ after $2 \mathrm{~h}$ of this reaction at room temperature. The persistently stirred reaction was carried out at room temperature overnight. Then, the synthetic HPEI nanogels were dialyzed in distilled water for 3 days. Subsequently, the HPEI nanogels were filtered by a syringe filter. The HPEI nanogels were adjusted to a final concentration of $1 \mathrm{mg} / \mathrm{ml}$ and stored at $4^{\circ} \mathrm{C}$ for future use.

Preparation of plasmid/HPEI complexes and transfection in vitro. The SKOV3 cell transfection was carried out using HPEI. To determine the optimal plasmid/HPEI ratio $(\mu \mathrm{g} / \mu \mathrm{g})$ for efficient gene delivery, we used the recombinant pVAX plasmid coding the green fluorescent protein (GFP) in a series of experiments with different plasmid/HPEI ratios transfecting SKOV3 cells in vitro. A maximum efficiency of transfection was obtained when $2 \mu \mathrm{g}$ plasmid/20 $\mu \mathrm{g}$ HPEI was used (data not shown).

The SKOV3 cells were seeded in 6-well plates at a density of $2 \times 10^{5} /$ well and incubated for $24 \mathrm{~h}$ to reach $80 \%$ confluence. Plasmid (pHSulf-1 or pEP, $2 \mu \mathrm{g}$ )/HPEI complexes $(20 \mu \mathrm{g})$ were prepared in $1 \mathrm{ml}$ DMEM medium without serum. DDP was prepared at a concentration of $5.0 \mu \mathrm{g} / \mathrm{ml}$. Normal saline (NS) was used as control. After the cells were incubated for $6 \mathrm{~h}$, the medium was replaced by $2 \mathrm{ml}$ DMEM supplemented with $10 \% \mathrm{FBS}$ and cultured for an additional $48 \mathrm{~h}$. The cells and the supernatants were collected for further assay. All transfections were performed in triplicate.

Detection of HSulf-1 mRNA expression by RT-PCR. The reverse transcription polymerase chain reaction (RT-PCR) was performed to detect the stable expression of HSulf-1 mRNA in transfected SKOV3 cells and intraperitoneal tumor tissues. The upstream primer and downstream primer for HSulf-1 were 5'-CGCGGATCCAAGATGAAGTATTCTTGCTGTGC-3' and 5'-CGCGATATCTTAACCTTCCCATCCATCCCATA-3', respectively. The total-RNA of each experimental group was extracted using TRIzol reagent (Invitrogen). The RT-PCR reactions with the isolated total-RNA $(0.5 \mu \mathrm{g})$ were carried out as follows: reverse transcription $\left(50^{\circ} \mathrm{C}, 30 \mathrm{~min}\right)$; denaturation $\left(94^{\circ} \mathrm{C}\right.$, $2 \mathrm{~min})$; amplification for 35 cycles $\left(94^{\circ} \mathrm{C}, 0.5 \mathrm{~min}\right)$, annealing $\left(60^{\circ} \mathrm{C}, 0.5 \mathrm{~min}\right)$ and extension $\left(72^{\circ} \mathrm{C}\right.$ for $\left.3 \mathrm{~min}\right)$; a terminal elongation procedure $\left(72^{\circ} \mathrm{C}, 10 \mathrm{~min}\right)$. After the reactions were completed, each RT-PCR product of $10 \mu \mathrm{l}$ was electrophoresed in a $1.0 \%$ agarose gel.

Western blot analysis. To confirm whether HSulf-1 was re-expressed in the transfected SKOV3 cells and intraperitoneal tumor tissues, the sample of each experimental group was lysed in modified RIPA lysis buffer containing $1 \mathrm{mM}$ PMSF. We used the BioRad protein assay (Bio-Rad, Hercules, CA) to detect the protein concentrations of lysates. Equal amounts of protein were 
loaded onto the $8 \%$ SDS-PAGE for electrophoresis and transferred to PVDF membranes (Millipore, Billerica, MA). Then, the blots were incubated with rabbit anti-human polyclonal antibody against HSulf-1 (diluted 1:800, Santa Cruz Biotechnology, Santa Cruz, CA) and the horseradish peroxidase-conjugated secondary antibody. The immunoreactive bands were detected by chemiluminescence. GAPDH was used as the internal standard.

Tumor model and treatment. The female athymic BALB/c nude mice (6-8 weeks old, 18-20 g each) were used to establish the intraperitoneal xenograft tumor model of human ovarian cancer as previously described (33). This animal experiment procedure was approved by the Institutional Animal Care and Treatment Committee of Sichuan University.

SKOV3 cell suspension ( $5 \times 10^{6}$ cells in $100 \mu$ l DMEM) was injected subcutaneously in the backs of 5 nude mice. When the diameter of s.c. tumors was approx. $1 \mathrm{~cm}$, tumors were harvested for i.p. inoculation. Seven days after i.p. tumor inoculation, 30 mice were randomly allocated into five groups (six mice/per group) to receive the following i.p. administration: i) untreated, normal saline (NS); ii) $5 \mu \mathrm{g}$ pEP/50 $\mu \mathrm{g}$ HPEI complexes, every two days for 12 times; iii) $5 \mu \mathrm{g}$ pHSulf-1/50 $\mu \mathrm{g}$ HPEI complexes, every two days 12 times; iv) DDP (3 mg/kg, Qinu Pharmacy Corporation, China), weekly for 3 times; v) combination therapy containing treatment of $5 \mu \mathrm{g}$ pHSulf-1/50 $\mu \mathrm{g}$ HPEI complexes for 12 times and DDP $(3 \mathrm{mg} / \mathrm{kg}$ ) for 3 times (volume $100 \mu \mathrm{l})$. All mice were sacrificed 3 days after the last injection and the intraperitoneal tumors were removed and weighed. When sacrificed, each mouse was observed and recorded in terms of ascites, and the number and location of peritoneally disseminated macroscopic tumors.

Immunohistochemistry staining. The intraperitoneal tumor tissues were fixed in $10 \%$ formalin ( $\mathrm{pH} 7.0)$, embedded in paraffin, and then cut into sections (3-5 $\mu \mathrm{m})$. The primary antibody of Ki-67 and CD31 immunostaining for intraperitoneal tumors is the rabbit anti-human Ki-67 antibody (diluted 1:100; Thermo Scientific, Hudson, NH) and goat anti-mouse CD31 (diluted 1:100; Santa Cruz Biotechnology), respectively. The tumor sections were deparaffinized and rehydrated at first. Heat-induced antigen retrieval was performed in $10 \mathrm{mM}$ citrate buffer ( $\mathrm{pH} \mathrm{6.0)}$ at $120^{\circ} \mathrm{C}$, and then endogenous peroxidase activity was blocked by $3 \% \mathrm{H}_{2} \mathrm{O}_{2}$. The sections were treated with $10 \%$ normal goat or rabbit serum to reduce non-specific staining, and then incubated with the primary antibody, biotinconjugated secondary antibody, streptavidin-biotin complex (SABC) successively. The immunoreaction was observed using diaminobenzidine (DAB) peroxide solution.

To quantify microvessel density (MVD), the sections were first scanned at x100 magnification to identify the areas having the highest vascular density without necrosis. The number of microvessels was counted in this areas at high-power field (hpf). In order to quantify the proliferation index, the percentage of $\mathrm{Ki}$-67-positive cells was observed in 10 random fields without necrosis at a magnification, $\mathrm{x} 400$.

Apoptotic analysis. Apoptosis in vivo was analysed using terminal deoxynucleotidyl transferase-mediated dUTP nick end-labeling (TUNEL) assay in accordance with the manufacturer's protocol (Promega, Madison, WI). Cell nuclei stained with dark green fluorescence were defined as TUNEL-positive and observed by fluorescence microscopy. The apoptosis index was analysed in 10 random fields without necrosis at x400 magnification.

Alginate-encapsulated tumor cell assay. SKOV3 ovarian tumor cells were resuspended in a $1.5 \%$ alginate (Sigma) solution, and then released into a solution of $250 \mathrm{mM} \mathrm{CaCl}_{2}$ to form the alginate bead. Alginate beads $\left(2 \times 10^{5}\right.$ cells/bead $)$ were embedded s.c. into both dorsal sides of the BALB/c nude mice under anesthesia (4 beads/mouse). Mice were treated with i.p. administration of $0.9 \%$ NS, pEP/HPEI complexes, pHSulf-1/HPEI complexes, DDP or pHSulf-1/HPEI complexes plus DDP, respectively. Treatment was carried out on the second day of implanting beads. The mice were injected intravenously with $100 \mu 1$ FITC-dextran (Sigma) $(100 \mathrm{mg} / \mathrm{kg}$ ) solution after 14 days. After FITC-dextran injection, alginate beads covering blood vessels were photographed after being exposed surgically and then quickly removed in $20 \mathrm{~min}$. The uptake of FITC-dextran was measured as described $(34,35)$.

Toxicity assessment. To evaluate the underlying side effects and toxicity of the combination therapy, the animal weight was monitored every four days and the correlative indices such as anorexia, diarrhea, skin ulceration and toxic death were monitored consecutively during the whole therapeutic procedure. The blood biochemical parameters such as ALT, AST, TBIL, ALB, BUN and CREA were detected to assess the potential side effects of the treatment at the end point of the experiment. Various organs (heart, liver, spleen, lung, kidney, brain, etc.) were harvested, fixed in $10 \%$ formalin $(\mathrm{pH} 7.0)$ and embedded in paraffin after sacrifice. Sections of these tissues (3-5 $\mu \mathrm{m})$ were stained with H\&E.

Statistical analysis. Comparisons of all numerical values among the different groups were performed using one-way analysis of variance (ANOVA) and the unpaired Student's t-test. A value of $\mathrm{P}<0.05$ was defined as statistically significant.

\section{Results}

Preparation and characterization of HPEI nanogels. As shown in Fig. 1A, in presence of EDC/NHS, the reaction between heparin and PEI2K was generated. When EDC/NHS activated heparin was dropped into PEI2K solution, one heparin molecule reacted with several PEI2K molecules and cross-linkage between heparin and PEI2K appeared. Consequently, the HPEI nanogels were formed through amide bonds.

Stable expression of HSulf- 1 in vitro. To examine whether the pHSulf-1/HPEI complexes resulted in expression of HSulf-1 in SKOV 3 ovarian cancer cells by transfection in vitro, SKOV3 cells were seeded in 6-well plates and incubated with NS, pEP/HPEI complexes, pHSulf-1/HPEI complexes, DDP or pHSulf-1/HPEI complexes plus DDP, respectively. Stable expression of HSulf-1 was detected using PT-PCR and western blot analysis after transfection for $48 \mathrm{~h}$. The HPEI nanogels efficiently transfected pHSulf-1 into SKOV3 cells in vitro and HSulf-1 could be expressed in SKOV3 cells after transfection with pHSulf-1/HPEI complexes, compared with control groups (Fig. 1B). 

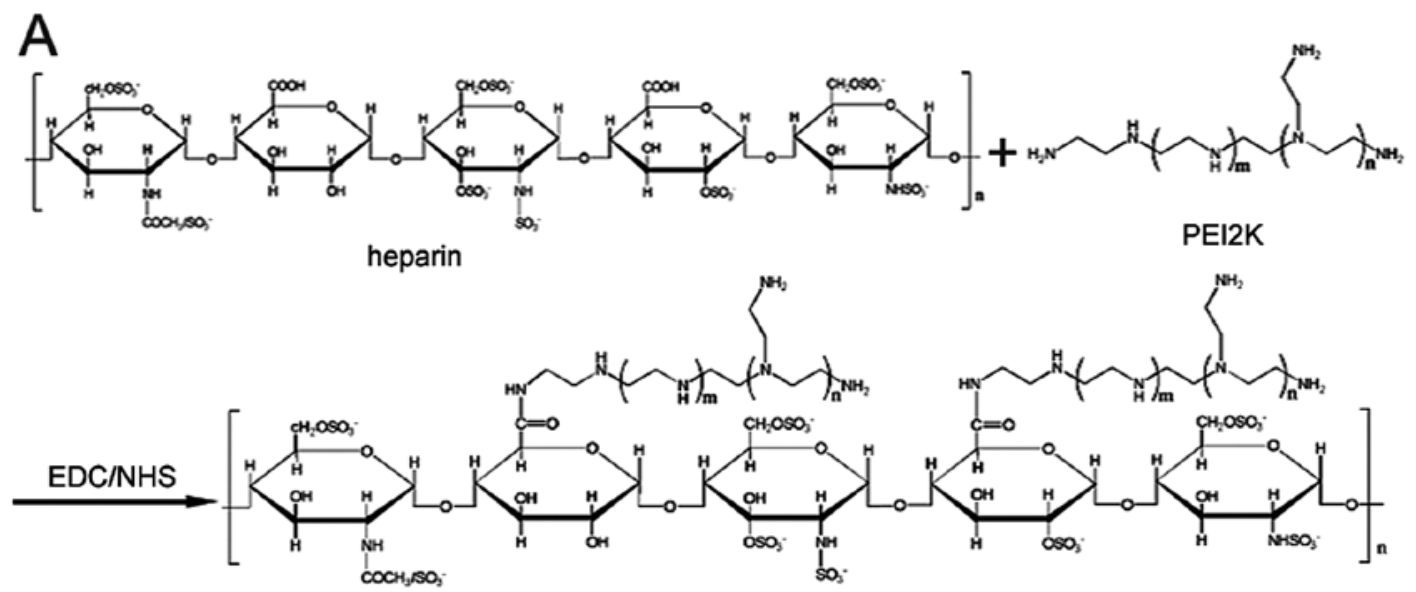

B $\begin{array}{llllll}\mathrm{M} & 1 & 2 & 3 & 4 & 5\end{array}$

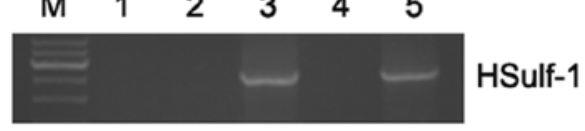

HPEl
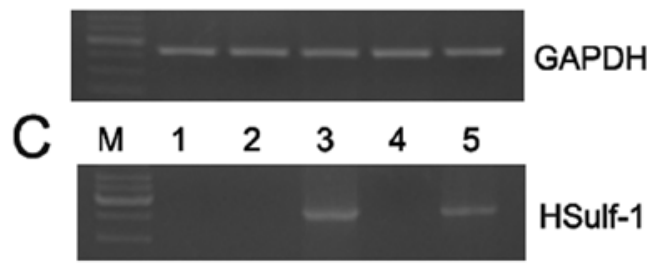

HSulf-1

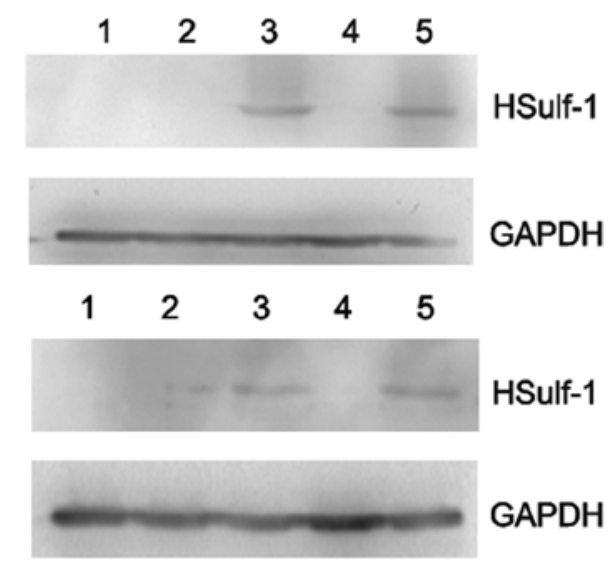

Figure 1. Synthesis of HPEI nanogels and expression of HSulf-1 in vitro and in vivo. (A) Preparative method of HPEI nanogels. Reaction between heparin and PEI2K was generated when catalyzed by EDC/NHS. (B) Identification of the expression of HSulf-1 in transfected SKOV3 cells in vitro by RT-PCR and western blot analysis. Lane 1, SKOV3 ovarian cancer cells were treated with NS; lane 2, pEP/HPEI complexes; lane 3, pHSulf-1/HPEI complexes; lane 4, DDP; or lane 5, pHSulf-1/HPEI plus DDP, respectively. Left, PT-PCR indicated only pHSulf-1/HPEI complexes treated cells had positive band of HSulf-1 (2,616 bp). GAPDH was used as the internal standard. Right, the result of western blot analysis showed a positive band ( 130 kDa) only occurred in cells transfected with pHSulf-1/HPEI complexes in contrast to control groups. (C) Expression of HSulf-1 in vivo. The intraperitoneal carcinomatosis model in nude mice was established and intraperitoneally administered with: lane 1, NS; lane 2, pEP/HPEI complexes; lane 3, pHSulf-1/HPEI complexes; lane 4, DDP; or lane 5, pHSulf-1/HPEI plus DDP, respectively $(80 \times 70 \mathrm{~mm}, 300 \mathrm{DPI})$.

Expression of HSulf-1 in vivo. An intraperitoneal xenograft model of human ovarian cancer was established to detect whether the pHSulf-1/HPEI complexes led to expression of HSulf-1 on SKOV3 cell line in vivo. The nude mice were injected with the above five agents, respectively. All mice were sacrificed after the last administration of 3 days and the intraperitoneal tumors were used for RT-PCR and western blot analysis. The pHSulf-1/HPEI complexes resulted in expression of HSulf-1 in vivo, but no expression of HSulf-1 could be examined in the three groups which were not treated with pHSulf-1/HPEI complexes (Fig. 1C).

Tumor suppressor function of the combination of HSulf-1 plus DDP. We established an intraperitoneal xenograft model of human ovarian cancer in nude mice to further investigate the efficacy of combination HSulf-1 with DDP in suppressing the growth of human ovarian tumor in vivo. As shown in Fig. 2, the group treated with pHSulf-1/HPEI or DDP alone exhibited significant inhibition of tumor growth (mean tumor weight, $0.38 \pm 0.19$ and $0.48 \pm 0.10 \mathrm{~g}$, respectively). The tumor growth of the two groups was significantly inhibited, compared with the group treated with NS and pEP/HPEI complexes (mean tumor weight, $1.62 \pm 0.36$ and $1.51 \pm 0.30 \mathrm{~g}$, respectively, $\mathrm{P}<0.01)$. However, the group of combination therapy exhibited an enhanced effect on tumor suppression, compared with the group treated with pHSulf-1/HPEI or DDP alone (mean tumor weight, $0.10 \pm 0.06 \mathrm{~g}, \mathrm{P}<0.01)$.

In the NS and pEP/HPEI groups, each mouse developed intraperitoneally macroscopic disseminated tumor nodules and a portion of them emerged bloody ascites (Table I). Microscopic examination verified that livers of three and four of the six mice developed tumor invasion, and the hemorrhagic ascites emerged in two and three of the six mice in pEP/HPEI and NS group, respectively. In the other three groups, the intraperitoneal tumor nodules were limited to the pelvis. No tissues and organs were obviously invaded by tumor and no ascites were found in these mice. One of the six mice in the group of combination therapy had no macroscopic tumor when sacrificed.

Inhibition of tumor cell proliferation in vivo. Ki-67 immunostaining was used to evaluate the proportion of proliferative 
Table I. Characterization of intraperitoneal xenografs of human ovarian cancer in different treatment group in nude mice (100x40 mm, 300 DPI).

\begin{tabular}{lcccc}
\hline $\begin{array}{l}\text { Treatment } \\
(\mathrm{n}=6)\end{array}$ & $\begin{array}{c}\text { Number } \\
\text { of nodules }\end{array}$ & $\begin{array}{c}\text { Mean weight } \\
(\mathrm{g}, \text { mean } \pm \mathrm{SD})\end{array}$ & $\begin{array}{c}\text { Liver invaded } \\
\text { by tumors }\end{array}$ & Ascites \\
\hline NS & $8 \pm 2$ & $1.62 \pm 0.36$ & 4 & 3 \\
pEP/HPEI & $6 \pm 2$ & $1.51 \pm 0.30$ & 3 & 2 \\
pHSulf-1/HPEI & $3 \pm 1$ & $0.38 \pm 0.19$ & 0 & 0 \\
DDP & $3 \pm 1$ & $0.48 \pm 0.10$ & 0 & 0 \\
pHSulf-1/HPEI+DDP & $2 \pm 1$ & $0.10 \pm 0.06$ & 0 & 0 \\
\hline
\end{tabular}

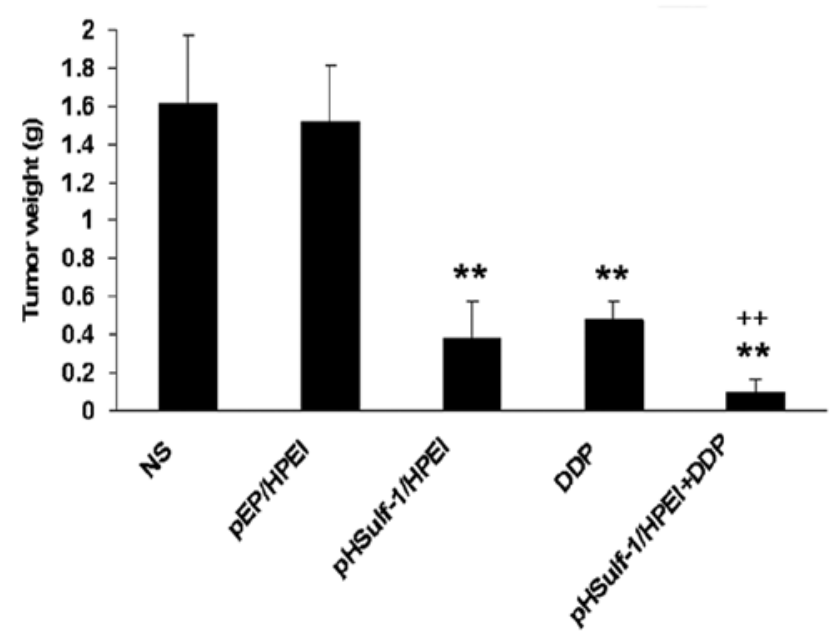

Figure 2. Tumor weights in the intraperitoneal xenograft model of human ovarian cancer in nude mice. The mice were intraperitoneally administered with NS, pEP/HPEI complexes, pHSulf-1/HPEI complexes, DDP or pHSulf-1/HPEI plus DDP, respectively. The result indicated that HSulf-1 exhibited significant efficacy in inhibiting tumor growth, but the combination of pHSulf-1/HPEI complexes with DDP displayed enhanced antitumor activity, compared with the therapy of HSulf-1 or DDP alone. Data are shown as means $\pm \mathrm{SE} .{ }^{* *} \mathrm{P}<0.01$ versus NS group; ${ }^{++} \mathrm{P}<0.01$ versus pHSulf-1/HPEI group $(80 \times 70 \mathrm{~mm}, 300 \mathrm{DPI})$.

tumor cells in each treatment group (Fig. 3). The empty vector and NS group demonstrated significant tumor cell proliferation in vivo. Nuclear staining in tumor tissues of $\mathrm{pHSulf-1/ \textrm {HPEI }}$ and DDP single therapy groups showed less staining for Ki-67 compared with the NS and pEP/HPEI groups $(\mathrm{P}<0.01)$. The mean percentage of Ki-67 positive cells in the pHSulf-1/HPEI group was not significantly different from that of DDP group $(\mathrm{P}>0.05)$. The cell proliferation dramatically decreased in tumors treated with pHSulf-1/HPEI plus DDP compared with control groups $(\mathrm{P}<0.01)$.

Induction of apoptosis in vivo. The pHSulf-1/HPEI and DDP monotherapy resulted in a significant increase of apoptotic tumor cells compared with the NS and pEP/HPEI treated groups $(\mathrm{P}<0.01)$, respectively. No significant difference was detected in the percentage of apoptotic cells between the two groups $(\mathrm{P}>0.05)$. The tumor tissues of the combination therapy group showed increased positive nuclei compared with the pHSulf-1/HPEI and DDP monotherapy groups $(\mathrm{P}<0.01)$. However, positive nuclei were rare in tumor tissues of the empty vector and NS groups, as shown in Fig. 3.
Inhibition of angiogenesis in vivo. Angiogenesis in tumor tissues was estimated by immunostaining using CD31 antibody which had high specific affinity for vascular endothelial cells. A significant reduction of MVD could be observed in pHSulf-1/HPEI and DDP treated groups compared with that in NS and $\mathrm{pEP} / \mathrm{HPEI}$ treated groups $(\mathrm{P}<0.01 ; \mathrm{Fig}$. 4). The MVD decreased more obviously in tumors of combination therapy group compared with $\mathrm{NS}$ and $\mathrm{pEP} / \mathrm{HPEI}$ groups $(\mathrm{P}<0.01)$. The capability of antiangiogenesis was also evaluated by alginate-encapsulated tumor cell assay and the quantification of the FITC-dextran uptake (Fig. 4). New blood vessels in alginate beads from the mice treated with pHSulf-1/HPEI and DDP were sparse, but twisted and rich blood vessels could be observed in alginate beads of NS and $\mathrm{pEP} / \mathrm{HPEI}$ treated groups. Blood vessels were obviously few in alginate beads of pHSulf-1/HPEI plus DDP group. In addition, the results of FITC-dextran uptake were similar to what we observed in alginate beads of different group.

Toxicity observation. The animal weight, considered as a parameter for evaluating anorexia, physical status or cachexia, was monitored every four days. There was a minor decrease of the body weights in the DDP and pHSulf-1/HPEI plus DDP groups, and no apparent differences were found between the two groups. Meanwhile, no significant differences in body weight were observed among the five groups ( $\mathrm{P}>0.05$, Fig. 5). In addition, no gross changes such as abnormal behavior and ruffling of fur were detected. The results of blood biochemical parameters and $\mathrm{H} \& \mathrm{E}$ histological staining indicated that there were no obvious pathological and biochemical alterations in various important organs.

\section{Discussion}

HSulf-1, a heparin-degrading extracellular endosulfatase which could selectively remove 6-O-sulfate from heparan sulfate is downregulated in the majority of examined cancer cell lines and it is markedly diminished or undetectable in $\sim 75 \%$ of ovarian cancers (7). Re-expression of HSulf-1 diminishes signaling of various heparin-binding growth factors, decreases cell proliferation, invasion and enhances drug-induced apoptosis in vitro (7-9,13). Some studies also showed that HSulf-1 could inhibit tumorigenesis and angiogenesis, and promote drug-induced apoptosis in vivo (10-12). The contribution of HSulf-1 to growth factor signaling and its effects on human cancerigenesis are under intensive investigation. In our previous study, we demonstrated that HSulf-1 could effectively inhibit intraperitoneal 

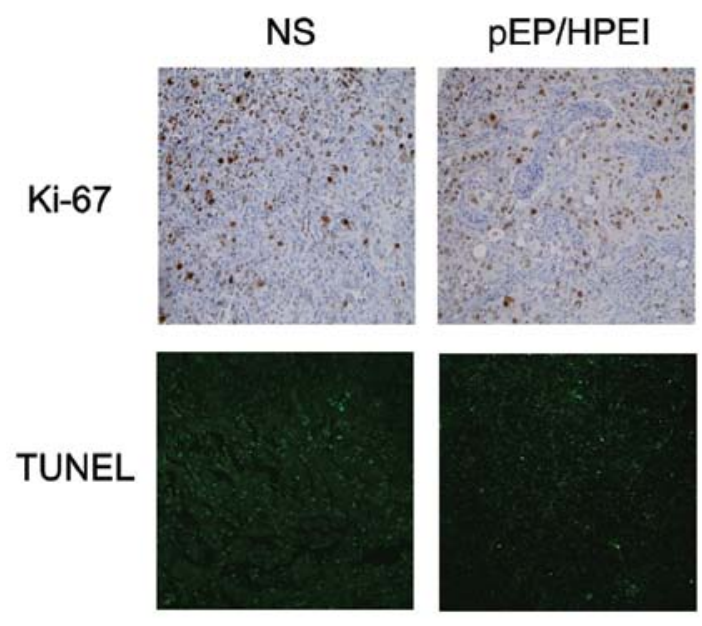

A

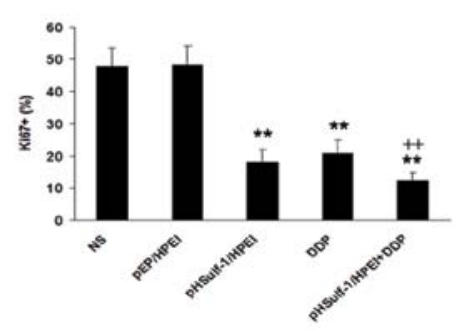

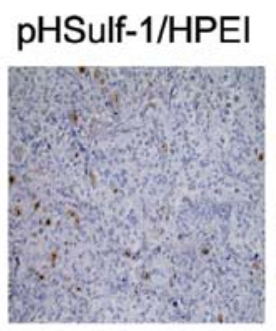

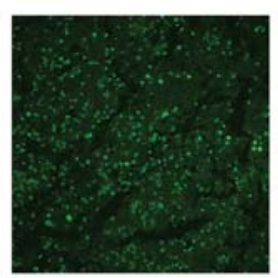

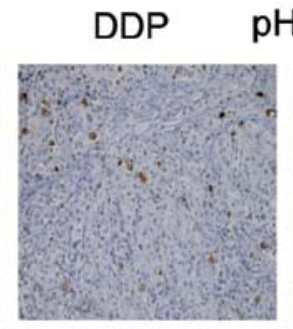

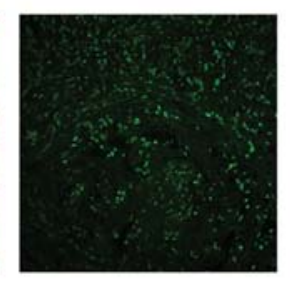

B

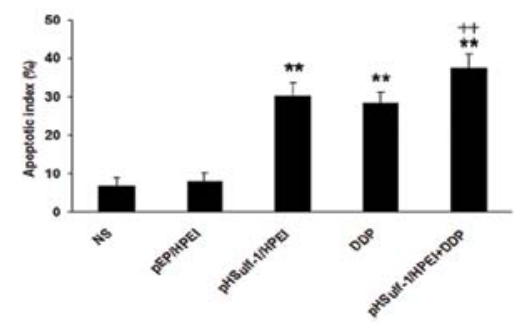

Figure 3. Effects of the different treatments on cell proliferation and apoptosis in vivo. (A) Significant suppression of cell proliferation was detected in the pHSulf-1/HPEI and DDP monotherapy groups. Many Ki-67-positive cells were identified in the NS and pEP/HPEI groups, but only a few positive nuclei occurred in pHSulf-1/HPEI plus DDP group; magnification, x200. (B) Apoptotic cells were observed in tumors treated with pHSulf-1/HPEI or DDP alone. Combination therapy obviously increased the percentage of apoptotic cells versus controls. Data are expressed as means \pm SE. ${ }^{* *} \mathrm{P}<0.01$ versus NS group; ${ }^{++} \mathrm{P}<0.01$ versus $\mathrm{pHSulf}-1 / \mathrm{HPEI}$ group; magnification, $\mathrm{x} 200(155 \times 100 \mathrm{~mm}, 300 \mathrm{DPI})$.

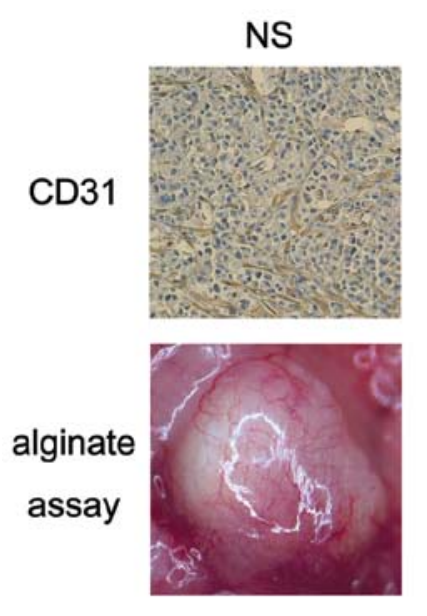

A
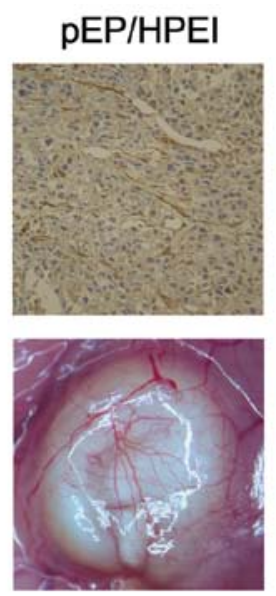
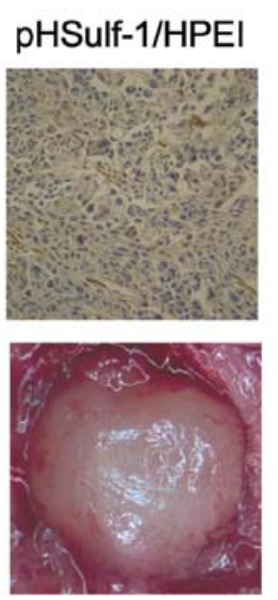
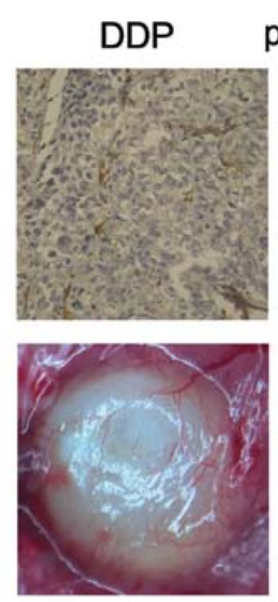

B
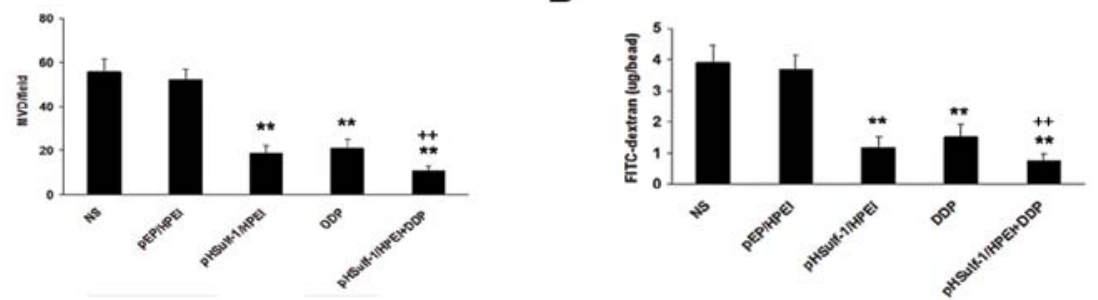

Figure 4. Effect on tumor angiogenesis was determined by CD31 immunostaining and alginate-encapsulated tumor cell assay in vivo. (A) CD31 immunostaining revealed that there were sparse microvessels in the tumor tissues of the pHSulf-1/HPEI and DDP monotherapy groups, and very few blood vessels in the combination therapy group; magnification, x400. (B) Alginate beads containing SKOV3 ovarian tumor cells, $2 \times 10^{5}$ cells/per bead, were implanted s.c. into the backs of 10 nude mice. Mice were intraperitoneally administered with NS, pEP/HPEI complexes, pHSulf-1/HPEI complexes, DDP or pHSulf-1/HPEI plus DDP. Beads were surgically removed and photographed. The suppression of angiogenesis occurred in the pHSulf-1/HPEI and DDP monotherapy groups. The function of anti-angiogenesis was more powerful in the combination therapy group. FITC-dextran uptake of beads from the different group revealed similar result. Data are expressed as means $\pm \mathrm{SE} .{ }^{* *} \mathrm{P}<0.01$ versus NS group; ${ }^{++} \mathrm{P}<0.01$ versus $\mathrm{pHSulf}-1 / \mathrm{HPEI}$ group; $(155 \mathrm{x} 100 \mathrm{~mm}, 300 \mathrm{DPI})$. 


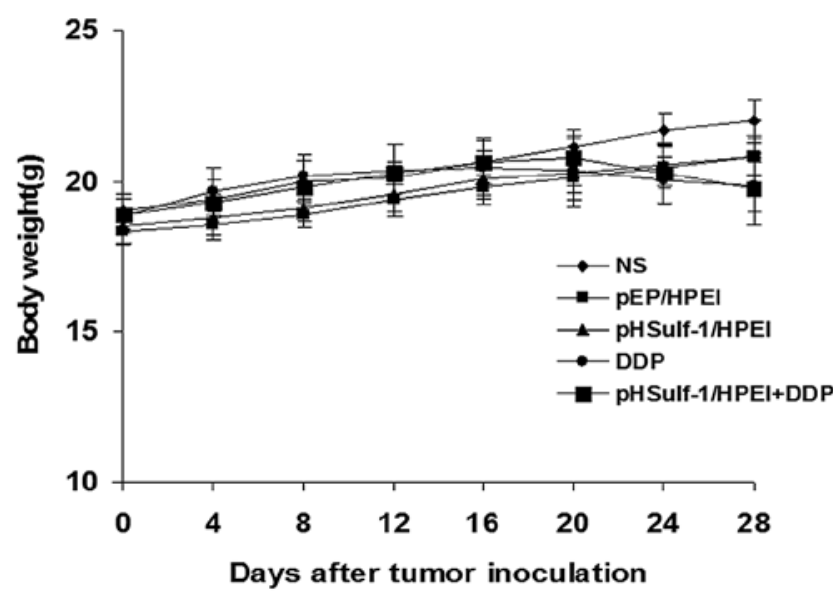

Figure 5. Lack of obvious toxicity-dependent weight loss in treated mice. There were no significant differences in body weight among the five groups $(\mathrm{P}>0.05)$, although minor weight loss occurred in the DDP and combination therapy groups. Data are expressed as means $\pm \mathrm{SD} ; \mathrm{n}=6$ for each group; $(80 \times 70 \mathrm{~mm}, 300 \mathrm{DPI})$

xenograft growth of human ovarian cancer (32). The findings that expression of HSulf-1 in cancer cells could inhibit tumorigenesis and tumor angiogenesis indicate that the modification of HSPG polysaccharide structure might be a reasonable approach for the therapy of cancer, in addition to, or in combination with conventional chemotherapy and antiangiogenic therapy.

Re-expression of HSulf-1 could sensitize the cancer cells to traditionally used chemotherapeutic agents such as cisplatin and paclitaxel (7-9,12-14). At present, cisplatin (DDP) has been widely used for the treatment of ovarian carcinoma. Severe side- effects and resistance to drug are common problems encountered in the procedure of cancer therapy, despite the significant efficacy of DDP in treating cancers. The therapy that combines conventional chemotherapeutic agents with inhibition of angiogenesis could have an additive effect on suppression of tumor growth and metastases in vivo (36). Therefore, in this study, we used the combination therapy of HSulf-1 plus DDP to develop more effective and novel therapeutic approach to treat ovarian cancer. Our finding indicated that HSulf-1 could inhibit tumorigenesis and angiogenesis in vivo, and the effect of HSulf-1 on ovarian cancer was markedly enhanced by combination with DDP. The antitumor, antiangiogenesis, antimetastasis, and antiapoptosis effects of HSulf-1 plus DDP were remarkably greater than those of HSulf-1 or DDP alone.

The mechanism of how this combination therapy exerts its effect is not clear. Angiogenesis, the development of new blood vessels from existing vasculature, is an essential factor for solid tumor growth and metastasis because beyond a critical extent, the tumor fails to expand further without neovascularization. The angiogenesis is controlled by the balance between various angiogenic and antiangiogenic agents $(37,38)$. HSulf- 1 may play a potential regulatory role in this multiple biological event, because heparan sulfate could interact with a great deal of these agents to enhance or inhibit their activities. HSulf-1 could modulate the function of heparan sulfate binding $\mathrm{VEGF}_{165}$ in proliferation and angiogenesis (11). The blockade of VEGF signaling can significantly enhance the efficacy of chemotherapeutic regimens (39). In this study, inhibition of angiogenesis by HSulf-1 was evaluated by CD31 and alginate-encapsulated tumor cell assay. Apparent decrease in tumor vessel formation was observed in the HSulf-1 treated group compared with the empty vector or NS treated group, indicating that HSulf-1 inhibited angiogenesis in vivo. This demonstrates that the intraperitoneal tumor might be exposed to an insufficient supply of oxygen and nutrients, which represses tumor growth, in agreement with the universally accepted concept that the growth of solid tumors such as breast and ovarian cancers depend on their capability of inducing tumor angiogenesis (40). In addition, angiogenesis in the HSulf-1 plus DDP group was more significantly suppressed compared with any other group. However, the exact antiangiogenic mechanism of this combination therapy remains unclear.

DDP is highly effective in the treatment of ovarian cancers. The biochemical mechanisms of DDP cytotoxicity involve binding of the drug to DNA and non-DNA targets, and the subsequent provocation of cell death through apoptosis, necrosis, or both (17). These antitumor mechanisms of DDP refer to interfering with DNA replication, forming lethal intrastrand DNA crosslinks, arresting cell cycle, and inducing cell apoptosis (18-20). In the present study, the antitumor and antiapoptosis effects were markedly enhanced by the combination therapy of HSulf-1 plus DDP, compared with the monotherapy of HSulf-1 or DDP. These results were consistent with the previous findings indicating that re-expression of HSulf-1 increased cisplatin-mediated cytotoxicity in ovarian cancer $(7,12,13)$. Further studies by Lai et al $(7)$ indicated that the typical biochemical hallmarks of apoptosis by the combination therapy of HSulf-1 plus DDP included cytochrome $c$ release from mitochondria and DNA fragmentation. HSulf-1 induced apoptosis by modulating the sensitivity of ovarian cancer cells to other stimuli, not by itself. Higher expression of HSulf-1 was related with somewhat higher induction of apoptosis.

With respect to the combination therapy, tumor growth might be influenced not only by direct cytotoxicity but also by inhibition of new vessel formation, and HSulf- 1 could enhance the antitumor effect of DDP, as well as decreasing development of drug resistance to DDP. On the other hand, the suppression of angiogenesis might lead to the death of tumor cells most distal to the established vasculature, thereby reducing tumor volume and facilitating access of the DDP throughout the tumor tissue.

Gene therapy, as a promising strategy to treat cancer, has achieved exciting progress in the past two decades. The development of safe and efficient gene carriers is one of the prerequisites for the successful application of gene therapy. The non-viral vectors, such as cationic lipids and polymers have many advantages over viral ones: low immunogenicity, easy to produce and no limitation to the size of transferred DNA molecules, but the toxicity is still a hindrance for the application of non-viral vectors in gene therapy $(21,23,24)$. Polyethylenimine (PEI), one of the most successful and widely studied gene delivery cationic polymers, is effective in gene delivery on account of its condensation of DNA, which facilitates endocytosis, and 'proton sponger' quality, which can prevent DNA from endosomal disruption $(25,31)$. Because PEI is not biodegradable and has the shortcoming of an association between transfection efficiency and cytotoxicity $(25,26)$, in our previous study, the low molecular weight PEI was conjugated chemically into biodegradable cationic nanogels by heparin, to develop a novel gene 
delivery vector $(31,32)$. HPEI nanogels were quickly degraded into low molecular weight PEI followed by excretion through urine in vivo and they also exhibited better blood compatibility, lower cytotoxicity, and stability in vitro, therefore, we used HPEI nanogels as the gene carrier in the present study.

Our data revealed that HPEI nanogels could efficiently deliver the HSulf-1 gene into SKOV3 ovarian cancer cells, and the expression of HSulf-1 in vitro and in vivo was detected at the mRNA and protein level. In this study, there were no apparent cytotoxicity and systemic toxic effects. Even though slight weight loss was observed in the DDP and HSulf-1 plus DDP-treated groups, it might be due to the toxic reaction of DDP. HSulf-1 delivered by HPEI nanogels exhibited an excellent tolerance in the procedure of intraperitoneal cancer treatment.

In conclusion, HSulf-1 shows potential for an effective antitumor capability against human ovarian carcinoma by way of inhibiting angiogenesis, reducing cell proliferation and inducing apoptosis. The antitumor activity of HSulf-1 was enhanced when combined with DDP. HPEI nanogels could serve as an efficient gene transfer vector, without apparent toxicity. Thus, HPEI nanogels delivering HSulf-1 combined with DDP might become a new and promising therapeutic strategy against human ovarian cancer.

\section{Acknowledgements}

This study was supported by the National Key Basic Research Program (973 Program) of China (2010CB529905 and 2011CB910703) and NIH81071862.

\section{References}

1. Shridhar V, Sen A, Chien J, Staub J, Avula R, Kovats S, Lee J, Lillie $\mathrm{J}$ and Smith DI: Identification of underexpressed genes in early- and late-stage primary ovarian tumors by suppression subtraction hybridization. Cancer Res 62: 262-270, 2002.

2. Morimoto-Tomita M, Uchimura K, Werb Z, Hemmerich S and Rosen SD: Cloning and characterization of two extracellular heparin-degrading endosulfatases in mice and humans. J Biol Chem 277: 49175-49185, 2002.

3. Ai X, Do AT, Kusche-Gullberg M, Lindahl U, Lu K and Emerson CP Jr: Substrate specificity and domain functions of extracellular heparan sulfate 6-O-endosulfatases, QSulf1 and QSulf2. J Biol Chem 281: 4969-4976, 2006.

4. Dhoot GK, Gustafsson MK, Ai X, Sun W, Standiford DM and Emerson CP Jr: Regulation of Wnt signaling and embryo patterning by an extracellular sulfatase. Science 293: 1663-1666, 2001.

5. Selva EM and Perrimon N: Role of heparan sulfate proteoglycans in cell signaling and cancer. Adv Cancer Res 83: 67-80, 2001.

6. Lin X: Functions of heparan sulfate proteoglycans in cell signaling during development. Development 131: 6009-6021, 2004.

7. Lai JP, Chien J, Staub J, Avula R, Greene EL, Matthews TA, Smith DI, Kaufmann SH, Roberts LR and Shridhar V: Loss of HSulf-1 up-regulates heparin-binding growth factor signaling in cancer. J Biol Chem 278: 23107-23117, 2003.

8. Lai JP, Chien J, Strome SE, Staub J, Montoya DP, Greene EL, Smith DI, Roberts LR and Shridhar V: HSulf-1 modulates HGF-mediated tumor cell invasion and signaling in head and neck squamous carcinoma. Oncogene 23: 1439-1447, 2004.

9. Lai JP, Chien JR, Moser DR, Staub JK, Aderca I, Montoya DP, Matthews TA, Nagorney DM, Cunningham JM, Smith DI, Greene EL, Shridhar V and Roberts LR: hSulf1 sulfatase promotes apoptosis of hepatocellular cancer cells by decreasing heparin-binding growth factor signaling. Gastroenterology 126: 231-248, 2004.
10. Dai Y, Yang Y, MacLeod V, Yue X, Rapraeger AC, Shriver Z, Venkataraman G, Sasisekharan R and Sanderson RD: HSulf-1 and HSulf-2 are potent inhibitors of myeloma tumor growth in vivo. J Biol Chem 280: 40066-40073, 2005.

11. Narita K, Staub J, Chien J, Meyer K, Bauer M, Friedl A, Ramakrishnan S and Shridhar V: HSulf-1 inhibits angiogenesis and tumorigenesis in vivo. Cancer Res 66: 6025-6032, 2006.

12. Liu P, Khurana A, Rattan R, Kalloger S, Dowdy S, Gilks B and Shridhar V: Regulation of HSulf-1 expression by variant hepatic nuclear factor 1 in ovarian cancer. Cancer Res 69: 4843-4850, 2009.

13. Staub J, Chien J, Pan Y, Qian X, Narita K, Aletti G, Roberts LR, Molina $J$ and Shridhar V: Epigenetic silencing of HSulf-1 in ovarian cancer: implications in chemoresistance. Oncogene 26: 4969-4978, 2007.

14. Lai JP, Sandhu DS, Shire AM and Roberts LR: The tumor suppressor function of human sulfatase 1 (SULF1) in carcinogenesis. J Gastrointest Cancer 39: 149-158, 2008.

15. Jemal A, Siegel R, Xu J and Ward E: Cancer statistics, 2010. CA Cancer J Clin 60: 277-300, 2010.

16. Ozols RF: Progress in ovarian cancer: an overview and perspective. EJC (Suppl) 1: 43-55, 2003.

17. Gonzalez VM, Fuertes MA, Alonso C and Perez JM: Is cisplatin-induced cell death always produced by apoptosis? Mol Pharmacol 59: 657-663, 2001.

18. Fuertes MA, Alonso C and Perez JM: Biochemical modulation of cisplatin mechanisms of action: enhancement of antitumor activity and circumvention of drug resistance. Chem Rev 103: 645-662, 2003

19. Reed E: Platinum-DNA adduct, nucleotide excision repair and platinum based anti-cancer chemotherapy. Cancer Treat Rev 24: 331-344, 1998

20. Cohen SM and Lippard SJ: Cisplatin: from DNA damage to cancer chemotherapy. Prog Nucleic Acid Res Mol Biol 67: 93-130, 2001

21. Edelstein ML, Abedi MR, Wixon J, Edelstein RM: Gene therapy clinical trials worldwide 1989-2004-an overview. J Gene Med 6: 597-602, 2004

22. Anderson DG, Peng W, Akinc A, Hossain N, Kohn A, Padera R, Langer R and Sawicki JA: A polymer library approach to suicide gene therapy for cancer. Proc Natl Acad Sci USA 101: 16028-16033, 2004.

23. Ferber D: Gene therapy. Safer and virus-free? Science 294: 1638-1642, 2001

24. Lv H, Zhang S, Wang B, Cui S and Yan J: Toxicity of cationic lipids and cationic polymers in gene delivery. J Control Release 114: 100-109, 2006.

25. Neu M, Fischer D and Kissel T: Recent advances in rational gene transfer vector design based on poly(ethyleneimine) and its derivatives. J Gene Med 7: 992-1009, 2005.

26. Kunath K, von Harpe A, Fischer D, Petersen H, Bickel U, Voigt $\mathrm{K}$ and Kissel T: Low-molecular-weight polyethyleneimine as a non-viral vector for DNA delivery: comparison of physicochemical properties, transfection efficiency and in vivo distribution with high-molecular-weight polyethyleneimine. J Control Release 89: 113-125, 2003.

27. Wen Y, Pan S, Luo X, Zhang X, Zhang W and Feng M: A biodegradable low molecular weight polyethyleneimine derivative as low toxicity and efficient gene vector. Bioconjug Chem 20: 322-332, 2009.

28. Gosselin MA, Guo W and Lee RJ: Efficient gene transfer using reversibly cross-linked low molecular weight polyethyleneimine. Bioconjug Chem 12: 989-994, 2001.

29. Forrest ML, Koerber JT and Pack DW: A degradable polyethyleneimine derivative with low toxicity for highly efficient gene delivery. Bioconjug Chem 14: 934-940, 2003.

30. Jeon O, Yang HS, Lee TJ and Kim BS: Heparin-conjugated polyethyleneimine for gene delivery. J Control Release 132: 236-242, 2008

31. Gou ML, Men K, Zhang J, Li YH, Song J, Luo S, Shi HS, Wen YJ, Guo G, Huang MJ, Zhao X, Qian ZY and Wei YQ: Efficient inhibition of C-26 colon carcinoma by VSVMP gene delivered by biodegradable cationic nanogel derived from polyethyleneimine. ACS Nano 4: 5573-5584, 2010.

32. Liu P, Gou ML, Yi T, Xie C, Qi XR, Zhou ST, Deng HX, Wei YQ and Zhao X: Efficient inhibition of an intraperitoneal xenograft model of human ovarian cancer by HSulf-1 gene delivered by biodegradable cationic heparin-polyethyleneimine nanogels. Oncol Rep 27: 363-370, 2012. 
33. Lin XJ, Chen XC, Wang L, Wei YQ, Kan B, Wen YJ, He X and Zhao X: Dynamic progression of an intraperitoneal xenograft model of human ovarian cancer and its potential for preclinical trials. J Exp Clin Cancer Res 26: 467-474, 2007.

34. Hoffmann J, Schirner M, Menrad A and Schneider MR: A highly sensitive model for quantification of in vivo tumor angiogenesis induced by alginate-encapsulated tumor cells. Cancer Res 57: 3847-3851, 1997.

35. He QM, Wei YQ, Tian L, Zhao X, Su JM, Yang L, Lu Y, Kan B, Lou YY, Huang MJ, Xiao F, Liu JY, Hu B, Luo F, Jiang Y, Wen YJ, Deng HX, Li J, Niu T and Yang JL: Inhibition of tumor growth with a vaccine based on xenogeneic homologous fibroblast growth factor receptor-1 in mice. J Biol Chem 278 21831-21836, 2003

36. Hu L, Hofmann J, Zaloudek C, Ferrara N, Hamilton T and Jaffe RB: Vascular endothelial growth factor immunoneutralization plus paclitaxel markedly reduces tumor burden and ascites in athymic mouse model of ovarian cancer. Am J Patho 161: 1917-1924, 2002.
37. Folkman J: What is the evidence that tumors are angiogenesis dependent? J Natl Cancer Inst 82: 4-6, 1990.

38. Carmeliet $\mathrm{P}$ and Jain RK: Angiogenesis in cancer and other diseases. Nature 407: 249-257, 2000

39. Klement G, Baruchel S, Rak J, Man S, Clark K, Hicklin DJ, Bohlen P and Kerbel RS: Continuous low-dose therapy with vinblastine and VEGF receptor-2 antibody induces sustained tumor regression without overt toxicity. J Clin Invest 105: R15-R24, 2000.

40. Neri D and Bicknell R: Tumour vascular targeting. Nat Rev Cancer 5: 436-446, 2005. 